\title{
The Analysis of Physical Decline belongs to the College Students from Gansu_based on the Sociological Perspective
}

\author{
Xiaodong $\mathrm{Yu}^{1, \mathrm{a}}$, Hexiang Jin ${ }^{1, \mathrm{~b}}$ \\ ${ }^{1}$ Northwest University for Nationalities, Lanzhou, Gansu, 730030 \\ ${ }^{a}$ email, ${ }^{b}$ email
}

\section{Keywords: College Students, Physical Decline, Influence Factor, Group Differences}

\begin{abstract}
The research purpose is through the students' diet, living habits, physical exercise, campus life and the environment of to find the sociological factors of college students' physical deterioration , and from the gender, nationality, urban and rural, only-child or not and family economic conditions to innovative the group differences. The research methods are questionnaire and $t$ interviews. Exercise results show that the reason mainly lies in the subjective consciousness of students, $81.5 \%$ of the cases attributed to exercise less, $79.8 \%$ due to unreasonable living habits, $65.4 \%$ due to poor diet, $23 \%$ due to pollution of the environment. $27.8 \%$ due to the lack of sports equipment and venues. Research has shown that the main factors affecting college students' physical deterioration are individual consciousness and school factor.
\end{abstract}

\section{Introduction}

Since the reform and opening up, China's rapid economic growth, people's lives have been greatly improved. However, under the background of the vigorous development of our socialist cause, the rapid improvement of economic strength and the improvement of people's material and cultural life, the physical fitness of college students have continued to decline. China's 19-year-old to 20-year-old college students' explosive force, strength, endurance and other physical quality decline year after year. According to the data released by the Ministry of Education in 2010, compared with 1985, 19-20 year-old college students decreased lung capacity by nearly $10 \%$, endurance, speed and other indicators continued to decline, overweight, obesity and myopia continues to increase. The decline in the quality of domestic college students in the first 10 years of this century compared to 2000 years ago, the data is more obvious, obesity continues to develop. One question in this survey: whether the decline in physical fitness is an objective fact from the student's subjective perspective, and about $70 \%$ of the sample supports this situation. University students are the main human resources of social development, is the backbone of the strategy of talent development in the backbone of its ability to shoulder the future successor of the motherland, must have a strong physique in order to better serve the motherland and the people, physical deterioration is bound Has caused serious obstacles to its development. The physical condition of college students is related to the factors such as nutrition, diet and physiological structure, but also by the change of the natural environment, especially the pollution of the environment, but more influenced by the social environment, such as the subjective consciousness of college students, School, society and other supervision and incentives related. Therefore, this problem can not simply analyze the reasons from a certain point of view, but to take into account the various aspects of the common impact of the mechanism, as well as different aspects of the interaction, so as to more comprehensive analysis of college students physical decline this objective fact. Although this study focuses only on the analysis of social factors, we still expect follow-up research to be multidisciplinary.

\section{Objects and Methods}

In this study, a total of 1,200 students from Northwest University for Nationalities, Lanzhou University, Gansu Polytechnic University and Gansu Agricultural University were investigated.

The research methods of this project are field research and quantitative analysis. A total of 1,200 students from four universities were surveyed by questionnaires. The sampling method mainly used 
stratified sampling in probabilistic sampling. Each of the four schools was assigned 300 places, each of the men and women, and the dormitory number was coded and simple random sampling was carried out. Effective questionnaires were 934 copies. At the same time, through the snowball sampling method to extract 100 students in-depth interviews to explore the underlying reasons behind the data. The data were analyzed by SPSS19.0 software, mainly using single factor analysis and two-factor chi-square analysis.

A total of 1,200 questionnaires and 934 valid questionnaires were sent to the survey. The survey subjects included a variety of professional, grade, gender, source, family economic situation, whether the only child, etc., has a good representation. Among the samples, boys accounted for $47.6 \%$, girls accounted for $52.4 \%$; freshman, sophomore accounted for $42 \%$ of the proportion of junior high school $14.8 \%$, senior students to return to the school rate is low, the minimum sample (4\%), but for In the case of the study, the grade difference is not significant and does not affect the results. Professional involved in law, finance, physics, materials, environment, medicine, a total of 50. The non-only child of the survey is two-thirds of the total. On the family economic situation, the variable is judged by the students subjective, but relative to the actual situation or uniform standards, there will be no excessive access from the data information point of view, was a normal distribution, concentrated in the medium economy, followed by To be well-off, wealth and poverty are relatively few. Investigation of the Han and ethnic minorities each accounted for half.

\section{Results}

A survey of breakfast intake showed that college students did not pay much attention to breakfast, and data showed that less than half of the students were able to eat breakfast every day, and those who sometimes eat or often do not eat breakfast, and often do not eat breakfast $15 \%$. Science shows that breakfast is very important for college students who are growing up, and the results are worrying. On the reasons for not eating breakfast on time, we made a return visit, interview only 60 people can guarantee a day to eat breakfast on time, do not eat breakfast for most of the reasons do not sleep late; no time too lazy downstairs, No time to eat; other reasons such as weight loss, economic difficulties and used to two meals a day. In the interview, as college students almost everyone knows the importance of breakfast on the human body, but still can not be achieved, which shows that young students, good health, it is easy to ignore the maintenance and self-maintenance of health, but also shows the students Their nutritional status is not enough attention or too confident.

Through the survey we can see that the lunch rate is relatively high, most students can achieve a daily lunch. Dinner is better than breakfast, but not lunch. In the type of food and mix, breakfast composition is healthy but most people pay more attention to lunch and dinner, especially lunch, dinner due to late get out of class, the school time is tense, girls lose weight without lunch attention. Overall, college students on the diet and nutrition with the degree of attention are not enough.

Evening sleep time, even if the evening class at 9:10 pm class, but before 10 o'clock to sleep only six people, the ratio of $0.6 \%, 10$ : $00-11: 00$ can sleep only $9 \%$, while the $11: 00$ after more than $90 \%$ of the sleep, of which 12:00 after sleeping about half of the number of sleep late to a large extent will affect the mental state of the next day, in the long run, the physical also have a negative impact.

The school is scheduled to take into account the need for nap, the morning class and afternoon class between about 2 hours, lunch and hour sleep time can be guaranteed, therefore, can rule out the short time factor. According to the interview we can see: 30 interviews with 20 people that clearly show that most of the time playing the phone. Nowadays, mobile phones are essential for college students, mobile phone information, video, games, chat is endless, ready to fill the blank time in life, playing mobile phone is a common problem in today's era, science and technology Of the developed so that the function of the phone can not be back, so reduce the time to play the phone can only rely on people's self-control. In addition to mobile phones, there are some students in the lunch break time to play the ball, to the library, chat, shopping, homework, in short, the importance of college students on the importance of lunch is not deep enough. 
College students sleep late, over $90 \%$ to 11 points after falling asleep, half of them after 12 o'clock to sleep, sleep time is not directly affect the breakfast and breakfast to eat. Sleep too late is a matter of concern. In this regard, we have a return visit to some students to do the interview to understand the reasons for the night, the return object is basically sleep late, the reason most play mobile phones, sleep, become a habit, there are some students because other people do not Sleep, he was also affected. From the survey point of view, many students are from the university began to sleep late, the other students before college sleep habits. It seems that the university does not have the control of the parents, there is no heavy work lead to fatigue, entirely by their own decision to rest, coupled with health awareness and self-control are not high, it is vulnerable to the impact of people around, it led to this group Sleep late, and almost everyone knows that night sleep on the next day a great impact on learning, but also did not want to stop this vicious circle.

Physical exercise is very important, but the active exercise of college students is not enough, from the data point of view, in a week $16 \%$ never exercise, $57 \%$ exercise 1,2 times, $20 \%$ exercise 3-4 times, 4 More than $7.5 \%$ above, the overall view of the degree of exercise is not high. Adhere to the regular exercise less, exercise the degree of attention caused by the body is not enough.

College students on their own physical condition of the evaluation, that their own good body only $11 \%$, most that better (54\%) and general (30\%), which shows that college students still have a clear understanding of their own, not blind Think it is very good, but the "general" ratio is higher than our imagination. In this regard, we conducted in-depth interviews in order to further understand the physical condition of students, the survey learned: many students think their body is not very good, the main problems are the following: 1 . waist, back, cervical pain; 2 . poor physical fitness, fatigue; 3. insomnia; 4. poor immunity, easy to get sick.

(68.8\%), do not like sports (46.3\%) and no time (45.3\%). The main reason for this is that the students' own subjective consciousness is not strong enough and their own ability to restrain themselves. Poor. The secondary reasons include no partner (34.9\%), no sports equipment (27.8\%), lack of space (19.2\%) and other objective reasons. This shows that college students believe that the main factors that impede the exercise is their own enthusiasm is not enough. Second, the school provides the venue and equipment and other hardware is also related.

From the perspective of college students, the main reason for the decline in physical fitness is less exercise (81.5\%), unhealthy habits (79.8\%) and unreasonable diet (65.4\%). Living habits mainly refers to late night, rest is not enough; eating habits have been done before the analysis. This shows that college students on the reasons for the decline in physical fitness are the right to know, but also in the improvement is not enough. Other causes such as heavy work burden (32.3\%), psychological stress (32.7\%), food insecurity (31.2\%) and serious environmental pollution (23\%).

For more than half (55.9\%) of the students who wish to participate in the school's competitive class activities, this data is worthy of an in-depth analysis. To this end, we have made in-depth interviews. Students are not enthusiastic about the activities of the school organization, the main reasons are the following: 1 . The school activities are not new, procrastination, waste of time, the game has shady, mainly the school level for the activities of the design disadvantage; Students do not have confidence in their own, self-feeling ability is not enough to participate will not have good results; 3 . like quiet, do not like poly heap; 4 . a waste of time, this activity to enhance self and future development does not make sense, Something else.

From the data of the school's propaganda, it is considered that the proportion of the benefits of physical education is less than $12 \%$, and that the proportion of propaganda is as high as $50 \%$ and that of publicity is almost 39\%. This shows that college students generally think that The school's efforts to promote physical exercise is not enough, which also reflects the side of the school's emphasis on physical exercise is not enough, should be more efforts in the publicity.

Although the burden of the university is less than that of the high school period, the learning pressure is still widespread. Most students think that the burden of study is normal. In the acceptable range, $18 \%$ think that the burden of study is very heavy, and only $10 \%$ think that the pressure is not great. From the data point of view, there is a certain period of school learning pressure, and this pressure will also affect the physical health of college students to some extent. 
For the school's physical fitness test, $41 \%$ of the students were able to actively participate in and support, but $51 \%$ said they did not advocate, $6 \%$ opposed. For further reasons, we have a part of the students had a return visit from the results of the interview to further see: support for physical fitness is mainly to enable students to have more understanding of their own physique; can urge exercise fitness, temper the will. For those who do not support physical fitness tests are: too formal, the type of test items and standards are too rigid; the schedule is too concentrated, most students only in the test before the assault training to cope with the examination.

Some schools will take such as morning punch cards, exercise scoring and other mandatory way to urge students to carry out physical exercise, for this form, students have different views: support attitude accounted for $30 \%, 70 \%$ of the opposition. This shows that the practice of the school on the one hand is too serious, not flexible enough, on the other hand, modern college students advocate free to arrange time, do not like to be bound, especially the mandatory activities.

In the case of active awareness is not strong, the school can be a sense of proportion of compulsory exercise to play a supervisory role, until the awareness of self-improvement, can be reduced, to give students the freedom to choose the space. Students are more likely to accept the school to provide and organize better sports facilities and sports activities, in addition, more publicity and education in some areas also have a certain role.

About $87 \%$ of students believe that environmental pollution is a serious problem, will affect the outdoor exercise, which shows that environmental pollution has become a factor in college students to reduce exercise. Environmental problems have been in Lanzhou, but in recent years haze spread to many of China's major cities, has become a serious social problem.

In addition to the above factors that affect the decline of college students' physical factors, there are differences between the different groups among college students, which directly affect the physical level. In this study, we have done this for further study, the criteria are divided into different types to analyze the differences between groups. To this end, we will be gender, ethnic origin and family economic situation as independent variables and physical conditions of the various variables to do cross-analysis and chi-square test, in order to be able to do more in-depth analysis of physical problems.

In the breakfast to eat, the girls eat better than boys (chi-square test significant degree of. = 0.006), girls pay more attention to breakfast than the boys eat and nutrition with breakfast. Lunch men and women no significant difference, while dinner eating boys is better than girls (sig. = 0.000).

From the data we can see that men and women in the amount of nap on the significant differences in girls' nap rate is higher (sig. $=0.000)$. About sleep time, half of the boys $(50.7 \%)$ are 12 o'clock after sleep, girls better, most of the 12 o'clock before, this shows that the boy's homemade ability worse, girls more regular habits. But the total amount of boys sleeps better than girls, which is the male's physiological structure or daytime consumption more relevant.

Exercise the body, the number of boys than girls exercise (sig. = 0.000). Body side performance boys than girls (sig. $=0.003$ ), which is related to gender differences in physical, boys sports ability is more prominent.

On the whole, girls living habits better than boys, and boys in the exercise of the body more active than girls. Diet and living habits on the girls to improve the physical role of play more, physical exercise on the boys to play a greater role in physical maintenance.

The proportion of the cultivars was changed from high to low. Visible, urban students pay more attention to the nutritional mix of breakfast, the relationship between the two variables in general is also more significant (sig. $=0.005$ ). Lunch and dinner are not very different, but still show the city than rural students eat more Hunxing, rural students to vegetarian-based more. City students are more nutritious and diverse in their diet choices. Dinner also shows this feature, lunch is not very different, urban students than rural students pay attention to the degree of nutrition with a slightly higher degree.

From sleep habits, rural students sleep more fixed and regular, usually between 6-8 hours (73.9\%), fall asleep earlier, while the city students are more discrete, less than 6 hours of sleep less 
and 10 hours more than the number of sleep more than rural students. Rural students sleep habits better than the city.

Rural students are significantly higher in urban or county (sig. $=0.000$ ) than in rural or rural areas. Rural students are more concerned about their physical condition, more attention to health.

Parents of the city are more active than rural parents in caring for children's exercise and eating and nutrition (sig. $=0.036$ ).

Rural children are more active in the competition and activities, and are more receptive to physical fitness tests and compulsory training.

According to the data analysis, the only child and non-only child in the following aspects there are significant differences: non-only child than the only child to do more housework (sig. $=0.000$ ); single child's parents of children's physical care and supervision (Sig. $=0.005$ ); non-only child is more receptive to the school exercise $($ sig. $=0.033$ ), indicating that non-only child self-reliance stronger, relatively more hard to endure,

According to the student's self-orientation, the questionnaire divides the family into four categories: affluence, well-off, middle and poverty. From the comprehensive data in all aspects, with the increase in family wealth, breakfast eating frequency gradually increased, and gradually focus on nutrition with (sig. $=0.02$ ). As the degree of affluence increases, the frequency of household chores is gradually reduced (sig. $=0.000$ ). In the physical test, the richer the family is, the worse the performance (sig. = 0.004). But the family is more wealthy, the better the feeling of self, which is different with the requirements of their own health standards, poor children need to do some housework and other physical activity, higher physical requirements, so that their physical fitness can not achieve the best status.

(Sig. $=0.000)$, which is more than the type of Han Chinese diet, ethnic minorities by the ethnic minorities, the number of ethnic minorities is higher than that of ethnic minorities. Religious and traditional diet effects, diet relative to a single. Minority college students are significantly higher than the Han nationality.

\section{Discussion}

From the above analysis, college students in physical maintenance there are a few more serious problems, if not seriously, the trend of physical decline will be difficult to change:

(A) diet nutrition is not up to standard, college students often do not eat breakfast, do not attach importance to nutrition with.

(B) sleep time is short, poor quality, late sleep late, living habits unhealthy.

(C) lack of exercise, lazy, no interest in after-school activities.

(D) indulge in the phone, computer, causing the eyes, cervical and many other discomfort.

(E) other issues, including depression and other mental health problems, food hygiene and environmental pollution is serious.

Can generally affect the decline in college students the main factors attributed to the following:

(1) personal factors: college students on their own physical condition is not enough attention, not be able to actively plan their own health work and rest, poor self-restraint, diet, sleep, exercise are more casual. In short, there are problems with the main initiative of college students in physical maintenance.

(2) family factors: many college students that their sleep habits are mainly in the family perennial formation, in addition, after the university, the parents of children care less than before, can not play a sufficient supervisory role, at the same time, The psychological care of the students, the family has more neglect, in the survey, some students have a serious psychological illness, parents know.

(3) school factors: First of all, the school on the unified physical exercise arrangements are not reasonable, can not improve the enthusiasm of the students exercise; Second, the physical test arrangements are not scientific; then, the school's physical health propaganda is not enough, so that students in the Their own understanding is not enough attention; Finally, there are other such as the school in the community and other activities of the design is not flexible enough, sports facilities is 
not enough, the problem of school pressure is also urgent to be resolved.

(4) social factors: such as food hygiene, environmental pollution and so on.

(5) other factors: gender factors in the living habits, exercise, diet and other aspects of the role; factors on the diet of nutrition, exercise and participate in activities have a certain impact; national factors on the diet greater impact; Women in the housework and other aspects of significant differences; family economic status of students' self-awareness have an impact.

Among them, personal factors and school factors as the most important.

Finally, to improve the physical condition of college students, but also rely on consciously, so that students recognize the importance of maintaining physical health. At the same time, schools should be committed to taking effective measures to improve the student's physique. As a macro-government should also be strict food hygiene standards, effective management of environmental pollution, for our future generations to create a better living environment.

\section{References}

[1] He Ying, Peng Xiaofeng. Value management research summary [J]. Finance and Accounting, 2005 (4): 110-113

[2] Ministry of Education. Ministry of Education on the 2010 national student physical and health research results announcement. Http: //www.moe.edu.cn/publicfiles/Business / htmlfiles / moe / s5948 / 201109 / 124202.html, 2011-08-29

[3] Yang Chuanchuan. Sociological analysis of college students' physical decline [J]. Journal of Sports Culture, 2015 (8): 145-147

[4] Jing Huifeng. 2004-2013 years of college students in Beijing physical fitness trends [J]. Occupation and Health, 2015, 31 (9): 1242-1244 